\title{
CME Tropical Medicine (123847): self-assessment questionnaire
}

\author{
Edited by Tahseen A Chowdhury
}

SAQs and answers are ONLINE for RCP fellows and collegiate members

\section{Format}

Candidates are asked to choose the best answer from the five possible answers. This best of five format is used in many medical examinations; however, the questions are not intended to be representative of those used in the MRCP(UK) Part 1 or Part 2 Written Examinations.

The answering process

1 Go to www.rcplondon.ac.uk/SAQ

2 Log on using your usual RCP username and password

3 Select the relevant CME question paper

4 Answer all 10 questions by selecting the best answer from the options provided

5 Once you have answered all the questions, click on Submit

\section{Registering your external CPD credits}

Carrying out this activity allows you to claim two external CPD credits. These will be automatically transferred to your CPD diary, where you can review the activity and claim your points.

1. Which one of the following is the principal vector for dengue globally?
(a) Aedes aegypti
(b) Aedes albopictus
(c) Anopheles gambiae
(d) Culex quinquefasciatus
(e) Haemagogus tropicalis

2. Concerning West Nile virus (WNV), which one of the following statements is true?
(a) Aedes mosquitoes transmit WNV
(b) Flaccid paralysis can be a feature of neuroinvasive WNV
(c) Roe deer are an important WNV reservoir
(d) WNV causes severe disease in $80 \%$ of infections
(e) Young adults are most vulnerable to severe WNV

3. Which of the following species of malaria is associated with the majority of deaths, both globally and in the UK?
(a) Plasmodium falciparum
(b) Plasmodium knowlesi
(c) Plasmodium malariae
(d) Plasmodium ovale
(e) Plasmodium vivax

4. A 34-year-old male attended the emergency department having returned from Burkina Faso 5 days previously. He was drowsy with a Glasgow coma score of 10. Temperature was $38.9^{\circ} \mathrm{C}$, blood pressure $100 / 60 \mathrm{mmHg}$ and respiratory rate 26 breaths per minute. Bloods showed haemoglobin of $109 \mathrm{~g} / \mathrm{dL}$, platelets of $93 \times 10^{9} / \mathrm{L}$, white cell count of $2.8 \times 10^{9} / \mathrm{L}$, creatinine of $183 \mu \mathrm{mol} / \mathrm{L}$, $\mathrm{PO}_{2}$ (on air) $9.5 \mathrm{kPa}$ and $\mathrm{pH}$ 7.25. Blood film showed early and late trophozoites of $P$ falciparum with $12 \%$ of red blood cells parasitised.

\section{What antimalarial should he receive?}
(a) Atovaquone-proguanil (Malarone ${ }^{\circledR}$ )
(b) Dihydroartemsinin-piperaquine $\left(\right.$ Euartesim $^{\circledR}$ )
(c) Intravenous quinine
(d) Intravenous artesunate
(e) Tafenoquine

5. Which of the following is a potential sign of severe malaria in adults?
(a) Glasgow coma score of 10
(b) Haemoglobin $10.1 \mathrm{~g} / \mathrm{dL}$
(c) Parasite count $1.3 \%$ of red cells parasitised
(d) Platelets below $90 \times 10^{9} / \mathrm{L}$
(e) Temperature $>38.7^{\circ} \mathrm{C}$

6. Which one of the following has an effective available vaccine?
(a) Crimean-Congo haemorrhagic fever
(b) Ebola virus
(c) Lassa fever
(d) Middle East respiratory syndrome coronavirus (Co-V)
(e) Zika virus

7. Which one of the following has improved the ability to offer good basic clinical care to patients during outbreaks of viral haemorrhagic fever?

(a) Evidence-based antiviral medications

(b) Inserting Wolbachia infection into mosquito populations to reduce transmission

(c) Locating isolation and treatment centres far from dense population centres

(d) Rapid near-patient biochemistry analysers

(e) Rapid transportation of samples to centralised laboratories 
8. Which one of the following arboviruses are transmitted by day-biting mosquitos?
(a) Crimean-Congo haemorrhagic fever
(b) Dengue
(c) Malaria
(d) Middle east respiratory syndrome Co-V
(e) Monkeypox

9. Which one of the following activity does not form part of the strategy for Guinea worm eradication?
(a) Containment of infected individuals
(b) Distribution of water filters
(c) Provision of safe drinking water
(d) Vaccination against Dracunculus medinensis
(e) Vector control of cyclops crustacean

10. Mass drug administration forms a part of the control strategy for many neglected tropical diseases.

Which one of the diseases has an alternative strategy proposed by World Health Organization?
(a) Echinococcosis
(b) Lymphatic filariasis
(c) Onchocerciasis
(d) Trachoma
(e) Yaws

\section{CME Medical Masterclass SAQ}

Answers to the CME SAQ published in Clinical Medicine in January 2019

$\begin{array}{llllllllll}\text { Q1 } & \text { Q2 } & \text { Q3 } & \text { Q4 } & \text { Q5 } & \text { Q6 } & \text { Q7 } & \text { Q8 } & \text { Q9 } & \text { Q10 } \\ \text { (a) } & \text { (e) } & \text { (e) } & \text { (a) } & \text { (d) } & \text { (d) } & \text { (e) } & \text { (d) } & \text { (b) } & \text { (c) }\end{array}$

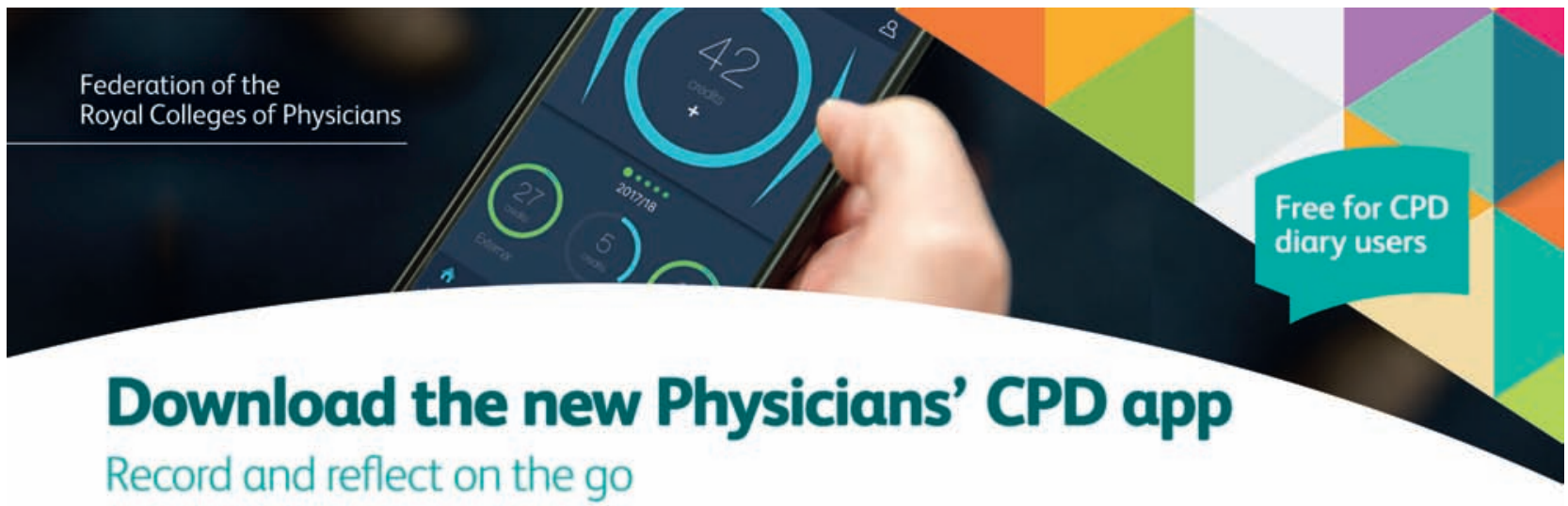

> Add and reflect on your educational activities, > Access our list of over 5,000 approved activities wherever you are - your changes will sync automatically with your online CPD diary*

$>$ Voice transcribe your development needs and reflections instantly

> Keep track of your CPD and monitor your progress to achieving your annual credit requirements

Simply download the Physicians' CPD app on your smartphone or tablet today and log in using your CPD diary account details. Now available on the IOS App Store and the Google Play Store

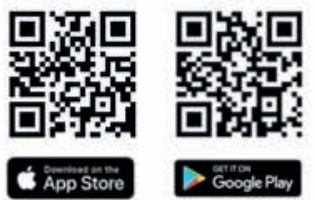

Physicians' CPD

https://cpd.rcplondon.ac.uk

"Registration to the CPD diary is required to use the Physicians' CPD app.
'An excellent and user-friendly app. I shall be using my CPD diary more frequently now!

Dr Andrew Lansdown, consultant endocrinologist 